\title{
Editorial
}

\section{Anticholinergic drugs and anaesthesia}

halothanc or a narcotic, although the narcotic used was not standardised.

The predominant cardiovascular effects of atropine and most other anticholinergic drugs are on heart rate and rhythm, with the main effect being an increase in the rate. Smaller doses may give rise to a small reduction in heart rate, but this is usually not observed following rapid intravenous administration. The degree of tachycardia produced depends upon the degree of pre-existing vagal tone; consequently healthy young adults who have a high resting vagal tone show the greatest increase in heart rate following atropine administration, whereas the effect in the very young and the elderly is relatively less. Administration of atropine, particularly by the intravenous routc, may also give rise to cardiac dysrhythmias such as nodal rhythm, ventricular ectopic beats and atrio-ventricular (A-V) dissociation.

The effects of anticholinergic drugs on heart or thythm may be greater in magnitude in anaesthetised than in conscious adults. ${ }^{1-3}$ This greater increase in heart rate in anaesthetised adults has been ascribed to the depression of vagal centres under anaesthesia. ${ }^{9}$ There is little information available regarding differences in the heart rate responses to anticholinergic agents in awake and anaesthetised children, although one study showed no difference between responses in awake children and those anaesthetised with halothane, ${ }^{5}$

In spite of their widespread use, it is not well known if the heart rate changes following administration of anticholinergic drugs differ during anaesthesia with different anaesthetic agents and techniques. The only available information is in children where greater increases in heart rate were observed during halothane anaesthesia in comparison with enflurane anaesthesia. ${ }^{6}$

The paper by Yamaguchi et al. ${ }^{7}$ in the current issue of the Journal sheds some light on this subject. These workers observed a greater increase in heart rate in adults anaesthetised with halothane, in comparison with enflurane, as had been observed previously in children. ${ }^{6}$ They also found the increases in heart rate after atropine administration to be similar in patients anaesthetiscd with

Dr. R.K. Mirakhur, Consultant Anaesthetist, Royal Victoria Hospital, Grosvenor Road, Belfast, Ireland, BT $126 \mathrm{BA}$
It is possible that halothane and enflurane may affect yagal tone to different extents. There is no direct evidence for this, although it was shown nearly twenty five years ago that halothane increased vagal tone. ${ }^{8}$ Similarly, administration of fentanyl has been shown to result in an increase in central vagal tone ${ }^{9}$ although it is not known if a single $100 \mu \mathrm{g}$ dose has such an effect. Buprenorphine has similar effects on heart rate, possibly due to a vagal effect once again. ${ }^{10}$

The depth of anaesthesia would affect the degree of vagal tone, which may then affect the heart rate response to atropine. It is difficult to judge the depth of anaesthesia prior to atropine administration in the patients studied by Yamuguchi et al. ${ }^{7}$ Whereas the concentrations of halothane and enflurane were reasonably close (approximately 4.0 MAC for halothane and 3.3 MAC for enflurane, both in 70 per cent nitrous oxide) one can only speculate about the comparative depth of anaesthesia following 100 $\mu \mathrm{g}$ of fentanyl and $0.2 \mathrm{mg}$ of buprenorphine. One would even doubt whether fentanyl $100 \mu \mathrm{g}$ and buprenorphine $0.2 \mathrm{mg}$ are equipotent doses. Yamaguchi et al. also did not find any correlation between the heart rates prior to atropine administration and the increase following it, as is observed in awake individuals. ${ }^{11}$ Could this be due to depressed ragal tone as a result of the anaesthetised state? The age span of the patients in this study was very wide, although the authors observed no correlation between age and the heart rate response. However we do not know if there was any correlation between the patients' ages and their basal heart rates.

An interesting aspect of the the study of Yamaguchi $e t$ $a l$. is the finding of no difference in the heart rate response to atropine between patients given a lumbar or a cervical epidural block. At face value these results would appear to be surprising, since it would be expected that the heart rate response in patients with a cervical epidural block would be greater, because of the cardiac sympathectomy, producing a vagotonic state. Yamaguchi et al. ${ }^{7}$ suggest that this is not so, because cervical epidural anaesthesia tlocks both the sympathetic as well as the parasympathetic innervation of heart. This explanation however is 
contrary to the common observation of a fall in heart rate after spinal and epidural analgesia, when the block extends higher than $T_{4}-T_{5}$ level. Is it possible that the results have been influenced by the administration of the light general anaesthesia? It is unfortunate that these authors did not report the heart rate and arterial pressure values after establishing the epidural blocks but before induction of general anaesthesia.

The findings from the study by Yamaguchi et al. ${ }^{7}$ and of other studies with anticholinergic agents have implications in clinical practice. Anticholinergic dnugs are used widely in anaesthesia for premedication, during surgery for prophylaxis and treatment of bradycardia and as adjuncts for the antagonism of neuromuscular block by anticholinesterases. Their use in premedication has been steadily decreasing. It is now widely accepted that omitting anticholinergic drugs from routine premedication has no adverse effects on the conduct of anaesthesia. ${ }^{12}$ Administration of such drugs may not only produce undesirable subjective effects, such as a very dry throat and loss of visual accommodation, but also give rise to cardiac dysrhythmias. ${ }^{13}$ The decreasing use of anticholinergic premedicants has been reflected in surveys conducted within the last ten years. ${ }^{14,15}$ The very young may however be considered an exception, but even in these patients a more predictable response can be obtained with intravenous administration.

Anticholinergic drugs are the mainstay for counteracting intraoperative bradycardias, paricularly those arising as a result of vagal stimulation. The study by Yamaguchi et al. ${ }^{7}$ has shown that cardiovascular changes in adults, particularly with regard to effects on heart rate, are much less during enflurane anaesthesia. Similar observations were made by Samra and Cohen in children. ${ }^{6}$ If limiting tachycardia produced by anticholinergic drug administration is the only aim during anaesthesia, enflurane would be the anaesthetic of choice. However this is rarely the case. It would also have been interesting to study the heart rate changes during isoflurane anaesthesia, since this agent is being used with ever increasing frequency. An alternative to the use of atropine for treating intranperative bradycardias is the use of the quaternary ammonium compound glycopyrrolate. ${ }^{16}$ Although the overall increase in heart rate with the two anticholinergic agents in anaesthetised patients is similar, the rate at which this occurs is slower with glycopyrrolate. ${ }^{17}$ This might avoid sudden shifts in the rate at which the vagal tone is altered in anaesthetised patients and thus prevent serious tachydysthythmias.

A situation where there is no controversy about the use of anticholinergic drugs is at the time of reversal of neuromuscular block. Anticholinergic drugs prevent the undesirable muscarinic effects of anticholinesterase drugs such as neostigmine, pyridostigmine or edrophonium. Atropine has long been used for this purpose, but while preventing neostigmine-induced decreases in heart rate, it gives rise to considerable initial increase in rate. This tachycardia may be minimised if enflurane is the inhalation anaesthetic used. Alternatively and mote convenient from a practical viewpoint, the fluctuations in heart rate can be reduced by substituting glycopyrrolate for atropine at the time of reversal of neuromuscular block. It has been shown that heart rates tend to remain much more stable with the use of this anticholinergic agent in a mixture with neostigmine, ${ }^{18-20}$ Glycopyrrolate would appear to be the agent of choice during halothane or fentanyl anaesthesia. The reasons for this cardiovascular stability are, once again, the slower onset of action of glycopyrrolate, matching a similar onset of action of neostigmine.

The cardiovascular sequelae of the reversal of neuromuscular block could be further reduced by using pyridostigmine or edrophonium as the anticholinesterase. The use of pyridostigmine is well established and it is believed to have a better safety margin than neostigmine. Pyridostigmine might also require a smaller dose of atropine or glycopyrrolate, but suffers from the disadvantage of a slow onset of action. ${ }^{21}$ Edrophonium, on the other hand, has been shown to have a rapid onset of action and requires only about half of the dose of anticholinergics in comparison with the doses used with neostigmine, atropine being the preferable agent in this case. ${ }^{21-23}$ This will obviously reduce the incidence and severity of fluctuations in heart rate. However, the biggest disadvantage of edrophonium is its inconsistency of action. ${ }^{24-27}$

In conclusion, anticholinergic agents exert significant effects on heart rate which may be exaggerated during general anaesthesia. Their use in routine premedication is unnecessary, although they must be used with anticholinesterases during reversal of non-depolarizing neuromuscular blockers. It is important to make the right choice of anticholinergic agents and anaesthetic techniques, particularly in patients with compromised cardiovascular systems.

\section{Les anticholinergiques et l'anesthésie}

Les effets cardiovasculaires prédominants de l'atropine et de la majorité des anticholinergiques s'exercent sur le rythme et la fréquence, la tachycardie êtant le problème majeur. Des plus petites doses peuvent amener une légère 
réduction de la fréquence cardiaque mais ceci n'est habituellement pas observé après l'administration intraveineuse rapide. Le degré de tachycardie produit dépend du tonus vagal pre-existant; ainsi des jeunes adultes sains ayant un tonus vagal élevé accuseraient une tachycardie plus importante après l'administration d'atropine alors qu'un effet moindre serait observé chez les très jeunes ou les gens âgés. L'administration d'atropine, particulièrement par voie intraveineuse, peut aussi occasionner des dysrythmies cardiaques tels qu'un rythme nodal, des extra-systoles ventriculaires et une dissociation auriculoventriculaire.

Les effets des anticholinergiques sur la fréquence cardiaque ou le rythme peuvent être plus importants chez les adultes anesthesiés comparativement à ceux qui sont conscients. ${ }^{1-3}$ Cette plus grande augmentation de la fréquence cardiaque chez les adultes anesthésiés a été attribuée à la dépression des centres vagues sous anesthésie. ${ }^{4}$ Il y a très peu d'information concemant les différences dans la réponse après anticholinergiques chez les enfants éveillés et anesthésiés. Une étude n'a cependant pas démontré de différence dans la réponse aux anticholinergiques chez les enfants éveillés comparativement à ceux anesthésiés avec l'balothane. ${ }^{5}$

Malgre leur utilisation généralisée, on ne sait pas si certe augmentation de la fréquence cardiaque après l'administration d'anticholinergiques diffère durant l'anesthésie avec les différents agents anesthésiques et les différentes techniques. La seule information disponible demeure celle observée chez les enfants qu'une plus grande augmentation de la fréquence cardiaque survient lors d'une anesthésie à l'halothane comparativement à l'anesthesie à l'enfluranc. ${ }^{6}$

L'étude de Yamaguchi et al. ${ }^{7}$ dans ce numéro du Journal nous éclaire quelques peu sur ce sujet. Ces chercheurs ont observé une plus grande augmentation de la fréquence cardiaque chez les adultes anesthésiés à l'halothane, comparativement à ceux anesthésiés à l'enflurane, comoborant ce qui a déjà été observé chez les entants. ${ }^{6}$ Ils ont aussi trouvé que l'agumentation de la fréquence cardiaque après l'administration d'atropine était similaire chez les patients anesthésiés à l'halothane aux narcotiques même si la technique aux narcotiques n'était pas standardisée.

Il est possible que l'halothane et l'enflurane affectent le tonus vagal à des degrés différents. Il n'y a aucune évidence directe à cet effet, même s'il a été démontré il y a déjà vingt ans que l'halothane augmente le tonus vagal. Aussi, il a été démontré que l'administration de fentanyl amène une augmentation du tonus vagal central ${ }^{9}$ mais 1 'on ne sait pas si une dose unique de $100 \mu \mathrm{g}$ aura cet effet. La buprénorphine a des effets similaires sur la fréquence cardiaque possiblement do à un effet vagal. ${ }^{10}$
La profondeur de l'anesthésic affecterait le degré du tonus vagal qui à son tour affecterait la fréquence cardiaque en réponse à l'atropine. Il est difficile de juger de la profondeur de l'anesthésie avant l'administration d'atropine chez les patients étudiés par Yamaguchi et $a l^{\text {? }}$ Alors que des concentrations d'halothane et d'enflurane étaient raisonnablement identiques (approximativement 4.0 MAC pour l'halothane et $3.3 \mathrm{MAC}$ pour l'enflurane, en présence de 70 pour cent de protoxyde d'azote) on ne peut que spéculer sur la profondeur de l'anesthésie après 100 $\mu \mathrm{g}$ de fentanyl et $0.2 \mathrm{mg}$ de buprénorphine. On peut même douter si les doses dc $100 \mu \mathrm{g}$ de fentanyl et de 0.2 mg de buprénorphine sont des doses equipotentes. Yamaguchi et al. n'ont pas trouvế de corrélation entre la fréquence cardiaque avant l'administration d'atropine et l'accroissement de la fréquence cardiaque qui s'cn cst suivie comme on en a observé chez les individus éveillés. ${ }^{11}$ Ceci serait-il dû à la dépression du tonus vagal amené par l'anesthésie? L'éventail des âges des patjents dans cette ćtude était très grand mème si les auteurs n'ont pas observé de corrélation entre l'âge et la réponse chronotrope. On ne sait pas cependant s'il y avait une comélation entre l'âge des patients et la fréquence cardiaque de base.

Un aspect intéressant de l'étude de Yamaguchi el al, est le fait qu'il n'y avait aucune différence dans la réponse chronotrope suite à l'atropine entre les patients ayant reçu un bloc epidural lombaire ou cervical. Pris comme tel, ces résultats seraient surprenants étant donné qu'on s'attend à ce que la réponse de la fréquence cardiaque chez les patients ayant subi un bloc epidural cervical serait plus grande a cause de la sympathectomie cardiaque produisant un état vagotonique. Yamaguchi $e t a l^{7}$ suggèrent qu'il n'en est pas ainsi car l'anesthésie épidurale cervicale bloquerait tant l'innervation sympathique que parasympathique du coeur. Cecte explication est cependant contraire à l'observation fréquente d'une bradycardie après l'analgésie épidurale ou rachidienne quand le bloc s'étend plus haut que $T_{4}-T_{5}$. Serait-ce possible que ces résultats soient influencés par l'administration d'une anesthésie générale légère? Il est malheureux que les auteurs n'aient pas rapporté les valeurs de la pression artérielle et de la fréquence cardiaque après l'établissement des blocs épiduraux alors qu'ils l'ont fait avant l'induction de l'anesthésie générale.

Les résultats de l'étude de Yamaguchi et al. ainsi quc d'autres études sur les agents anticholénergiques ont des implications cliniques pratiques. Les anticholinergiques sont utilisés communément en anesthésie pour la prémédication, lors de la chirurgie afin de prévenir et de traiter les bradycardies et avec les anticholinestérasiques afin d'antagoniser les blocs neuromusculaires. Leur utilisation en prémédication est en train de diminuer progressive- 
ment. Il est actuellement accepté que l'omission des drogues anticholinergiques de la prémédication de routine n'a aucun effet secondaire sur la conduite de l'anesthésie. ${ }^{12}$ L'adninistration de tels médicaments peut non seulement produire des effets subjectifs indésirables telles que sécheresse de la gorge et perte d'accomodation visuelle, mais peut aussi provoquer des dysrythymies cardiaques. ${ }^{13} \mathrm{~L}$ 'utilisation décroissante des anticholincrgiques lors de la prémédication se réflète dans les enquêtes faites durant ces dix dernières années. ${ }^{14,15}$ Les très jeunes peuvent cependant être'considérés comme une exception car chez ces patients une réponse plus prévisible peut être abtenue avec l'administration intraveineuse.

Les médicaments anticholinergiques sont les piliers de la correction de bradycardie intra-opératoire particulièrement cellc survenant après stimulation vagalc. L'etude dc Yamaguchi et al. ${ }^{7}$ a démontré que les variations cardiovasculaires chez l'adulte, particulièrement les effets sur la fréquence cardiaque sont moindres lors de l'anesthésie à l'enflurane. Des observations similaires ont été faites par Samra et Cohen chez les enfants. ${ }^{6} \mathrm{Si}$ la limitation de la tachycardie produite par l'administration de médicaments anticholinergiques est le seul but lors de l'anesthésie, l'enflurane serait l'anesthésique de choix. Cependant ceci est rarement le cas. Il aurait été intéressant d'étudier les changements de la fréquence cardiaque lors de l'anesthésie à l'isoflurane étant donné que cet agent est de plus en plus employé. Comme altemative à l'utilisation de latropine pour le traitement des bradycardies intraopératoires on utilise le glycopyrolate, ${ }^{16}$ un ammonium quaternaire. Même si l'augmentation de la fréquence cardiaque avec ces agents anticholinergiques chez les patients anesthésiés est identique, celle-ci survient plus lentement avec le glycopyrrolate. ${ }^{17}$ Ceci peut éviter des changements rapides de la fréquence cardiaque chez les patients anesthésiés et ainsi prévenir des tachydysrythmies sérieuses.

Une situation où il n'y a aucune controverse sur l'utilisation des anticholinergiques serait lors de l'antagonisme du bloc neuromusculaire. Les anticholinergiques préviennent les effets muscariniques indésirables des anticholinestérasiques tels que la néostigmine, pyridostigmine ou l'édrophonium. L'atropine a été longtemps utilisée pour cette fin mais alors qu'elle prévenait la diminution de la fréquence cardiaque induite par la néostigmine, elle amenait une augmentation considérable initialement de la fréquence cardiaque. Cette tachycardie peut être diminuée si l'enflurane était l'agent anesthésique utilisé. Du point de vue pratique cette fluctuation de la frequence cardjaque peut être diminuée en substituant le glycopyrrolate à l'atropine lors de l'antagonisme du bloc neuromusculaire. Il a été démontré que la fréquence cardiaque tend à demeurer plus stable avec cet agent anticholinergique quand il est mélangé avec la néostig. mine. ${ }^{18-20}$ Le glycopyrrolate serait l'agent de choix durant l'anesthésie à l'halothane ou au fentanyl. Les raisons de cette stabilité cardiovasculaire seraient encore une fois dues au début d'action du glycopynolate qui serait similaire au début d'action de la néostigmine.

La séquelle cardiovasculaire de l'antagonisme du bloc neuromusculaire pourrait encore plus être réduite par l'utilisation de la pyridostigmine ou l'édrophonium comme anticholinestérasique. Cette utilisation de la pyridostigmine est bien établie et l'on croit qu'elle aurait une plus grande marge de sécunté que la néostigmine. La pyridostigmine pourrait aussi requérir une plus petite dose d'atropine ou de glycopyrrolate mais présenterait comme un désavantage un début d'action lent. ${ }^{21}$ L'édrophonium par contre, aurait un début d'action rapide et nécessiterait seulement la moitié de la dose d'anticholinergique comparativement aux doses utilisées avec la néostigmine, l'atropine étant l'agent de choix dans ce cas. ${ }^{21-23} \mathrm{Ceci}$ réduirait clairement l'incidence et la sévérité des fluctuations de la fréquence cardiaque. Cependant le désavantage majeur de l'édrophonium est l'inconsistance de son action. ${ }^{24-27}$

En conclusion, les agents anticholinergiques exercent des effets significatifs sur la fréquence cardiaque pouvant être exagérés lors de l'anesthésie générale. Leur utilisation de routine en prémédication n'est pas nécessaire. Ils doivent cependant êttre utilisés avec les anticholinestérasiques lors de l'antagonisme des blocs musculaires non dépolarisants. Il est important de faire le bon choix dans les agents anticholinergiques et les techniques anesthésiques particulièrement chez les patients ayant un système cardiovasculaire compromis.

\section{References}

1 Jones RE, Deutsch S, Turndorf $H$. Effects of atropine on cardiac rhythm in conscious and ancsthetised man. Anesthesiology 1961; 22: 67-73.

2 Farman JV. Circulatory effects of atropine during halothane anaesthesia. Br J Anaesth 1967; 39: 226-35.

3 Eikard B, Andersen $J R$. Arthythmias during halothane anaesthesia. II: The influence of atropine. Acla Anaesthesiol Scand 1977; 21: 245-51.

4 Eger EI. Atropine, scopolamine and related compounds. Anesthesiology 1962; 23: 365-83.

5 Mirakhur $R K$, Jones $C J$ Atropine and glycopyrrolate: Changes in cardiac rate and rhythm in conscious and anaes. thetised children. Anaesth Intensive Care 1982; 10: 328-32.

6 Samra SK, Cahen PJ. Modification of chronotropic response to anticholinergics by halogenated anaesthetics in children. Can Anaesth Soc J 1980; 27: 540-5.

7 Yamaguchi $H$, Dohi $S$, Sato $S$, Naito $H$. Heart rate response to atropine in humans anaesthetised with five 
different techniques. Can J Anaesth. 1988; 35: 451-6.

8 Price $N L$, Linde $W$, Morse NT. Central nervous actions of halothane affecting the systemic circulation. Anesthesiology 1963; 24: 770-8.

9 Reitan JA, Stengert KB, Wymore ML, Martucci RW. Central vagal control of fentanyl-induced bradycardia during halothane anesthesia. Anesth Analg 1978; 57: 31-6.

10 Cowan A, Doxey JC, Harry EJR. The animal pharmacology of buprenorphine, an oripavine anaigesic agent. $\mathrm{Br} \mathrm{J}$ Pharmacol 1977; 60: 547-54.

11 Boba $A$. The effect of atropine on the heart rate. Can Anaesth Soe J 1976; 23: 92-8.

12 Mirakhw RK. Anticholincrgic drugs. Br J Anaesth 1979; 51: 67I-9.

13 Mirakhur RK, Clarke RSJ, Elliont J, Dundee JW. Alropine and g]ycopyrronium premedication. A comparison of the effects on cardiac rate and rhythm during the induction of anaesthesia. Anaesthesia 1978; 33: 906-12.

14 Mirakhur RK, Clarke RSJ, Dundee JW, MCDonald JR Anticholinergic drugs in anacsthesia. A survey of their present position. Anaesthesia 1978; 33: 133-8.

15 Lunn JN, Farrow SC, Fowkes FGR, Robertson IB, Samuel $P$. Epidemiology in anaesthesia I: Anaesthetic practice over 20 years. Br J Anaesth 1982; 54: 803-9.

16 Mirakhur RK, Dundee JW. Glycopyrrolate: Pharmacology and clinical use. Anaesthesia 1988; 38: 1195-1204.

17 Mirakhur RK, Jones CJ, Dundee JW. Effect of intravcnous administration of glycopyrrolate and atropine in anaesthetised patients. Anaesthesia 1980; 35: 277-81.

18 Ramamurthy S, Shaker $M H$, Winnie AP. Glycopyrrolate as a substitute for atropine in neostigmine reversal of muscle relaxant drugs. Can Anaesth Soc J 1972; 19: 399-411.

19 Mirakhur RK, Dundee JW, Clarke RSJ. Glycopystolateneostigmine mixture for antagonism of neuromuscular block: comparison with atropine-neostigmine mixture. $\mathrm{Br}$ ] Anaesth 1977; 49: 825-9.

20 Mirakhur RK, Dundee JW, Jones CJ, Coppel DL, Clarke RSI. Reversal of neuromuscular blockade: dose determination studies with atropine and glycopyrrolate given before or in a mixture with neostigmine. Anesth Analg 1981; 60: 557-62.

21 Cronnelly R, Morris RB, Miller RD. Edrophonium: Duration of action and atropine requirement in bumans during halothane anesthesia. Anesthesiology 1982; 57: 261-6.

22 Bevan $D R$. Reversal of pancuronium with edrophonium. Anaesthesia 1979; 34: 614-9.

23 Mirakhur $R K$. Antagonism of the muscarinic effects of edrophonium with atropine or g]ycopynolate: a comparative study. Br J Anaesth 1985; 57: 1213-6.
24 Lavery GG, Mirakhur RK, Gibson FM. A comparison of edrophonium and neostigminc for the antagonism of atracurium-induced neuromuscular block. Anesth Analg 1985; 64: 867-70.

25 Mirakhur RK, Gibson FM, Lavery GG. Antagonism of vecuronium-induced neuramuscular blockade with edrophonium or neostigmine. Br J Anaesth 1987; 59: 473-7.

26 Mirakhur $R K$. Antagonism of pancuronium and tubocurarine blocks by edrophotium or neostigmine: a comparative study. Eur J Anaesthesiol 1987; 4: 411-9.

27 Caldwell JE, Roberison EN, Baird WLM. Antaganism of vecuronium and atracurium: Comparison of neostigmine and edrophonium administered at $5 \%$ twitch height recovery. Br J Anaesth 1987; 59: 478-81. 